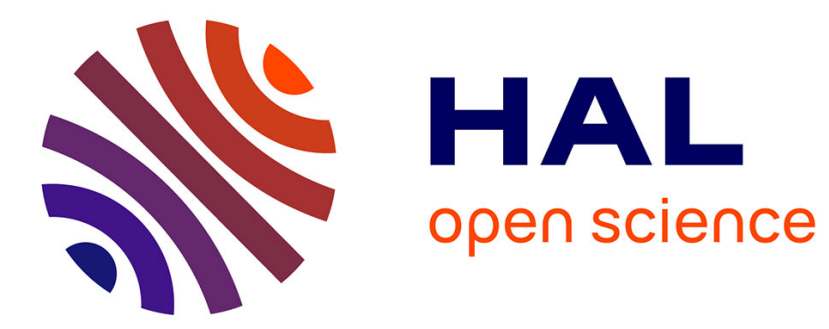

\title{
Dynamics of drop absorption by a swelling fiber
}

\author{
Pierre van de Velde, Suzie Protière, Camille Duprat
}

\section{To cite this version:}

Pierre van de Velde, Suzie Protière, Camille Duprat. Dynamics of drop absorption by a swelling fiber.

Soft Matter, 2021, 17 (25), pp.6168-6175. 10.1039/cXsm00000x/ . hal-03389048

\section{HAL Id: hal-03389048 \\ https://hal.sorbonne-universite.fr/hal-03389048}

Submitted on 20 Oct 2021

HAL is a multi-disciplinary open access archive for the deposit and dissemination of scientific research documents, whether they are published or not. The documents may come from teaching and research institutions in France or abroad, or from public or private research centers.
L'archive ouverte pluridisciplinaire HAL, est destinée au dépôt et à la diffusion de documents scientifiques de niveau recherche, publiés ou non, émanant des établissements d'enseignement et de recherche français ou étrangers, des laboratoires publics ou privés. 


\title{
Dynamics of drop absorption by a swelling fiber
}

\author{
Pierre Van de Velde, ${ }^{* a}$ Suzie Protière ${ }^{b}$, and Camille Duprat ${ }^{a}$
}

Received Date

Accepted Date

DOI: $00.0000 / x x x x x x x x x x$
Swelling of individual fibres exposed to a favorable solvent may affect the mechanical properties or shape of a fibrous material. We provide experimental results of the absorption dynamics of a single drop deposited on a swellable fibre and show that the total absorption time is highly dependent on the fibre geometry and drop volume. The curvature of the fibre prevents the total spreading of a drop even if the fluid is fully wetting the fibre. For drops larger than a critical volume, a local saturation of the fibre is thus reached below the drop, leading to a strong increase in the total absorption time. These observations are then rationalized with a simple pseudo-diffusive model to understand the drop absorption and fibre swelling dynamics. This minimal model provides quantitative predictions of the total absorption time.

\section{Introduction}

At the macroscopic scale, swelling can be viewed as the growth of a material during the imbibition of a fluid. This phenomenon has particularly strong implications in many processes where fibrous materials are involved. Fibre swelling can be observed during the fabrication of paper, where the swelling of cellulosic fibres in water affects several parts of the process and handling of paper 1 , or upon wetting of textiles. Most textiles are composed of several yarns, which are themselves formed of multiple monofilaments (fibres). Many of these fibres absorb moisture (e.g. cotton, viscose) which modifies the wicking kinetics in the yarns and induces deformations at the scale of the textile $\mathrm{e}^{2}$. Moreover, biosourced fibres such as cellulose or flax, which can be locally produced and readily available, and thus seem to be a promising option for garment production, construction and many other technical uses, strongly absorb solvents which leads to an alteration of their properties and shape once wetted or after drying.

Swelling dynamics is often studied through the growth of unconstrained elastomeric gels in a solvent ${ }^{3}$. However, the rate of swelling depends not only on the gel property but also on its geometry via the mechanics of swelling itself. For example, immersed spherical gels develop wrinkles and folds due to a rapid outer layer growing while the inner core is still constrained 4 , while spaghetti will grow and sag during hydration ${ }^{5}$. A common example of morphological changes induced by swelling is observed when a sheet of paper starts to bend when placed at the surface of water 6 . These large shape changes are due to the differential swelling occuring as the bottom layer in contact with water swells while the top layers remain dry and unswollen, until

\footnotetext{
${ }^{a}$ LadHyX, CNRS, Ecole Polytechnique, Institut Polytechnique de Paris, UMR 7646, 91128 Palaiseau, France, , E-mail: camille.duprat@ladhyx.polytechnique.fr

${ }^{b}$ Institut Jean le Rond J'Alembert, CNRS, Sorbonne UniversitÂl', UMR 7190, F-75005 Paris, France, Email: protiere@ida.upmc.fr

$\dagger$ Electronic Supplementary Information (ESI) available: [details of any supplementary information available should be included here]. See DOI: $10.1039 / \mathrm{cXsm00000x/}$

$\ddagger$ Additional footnotes to the title and authors can be included e.g. 'Present address:' or 'These authors contributed equally to this work' as above using the symbols: $\ddagger, \S$, and $\uparrow$. Please place the appropriate symbol next to the author's name and include a $\backslash$ footnotetext entry in the the correct place in the list.
}

the swelling front diffuses to the top of the sheet. When studying the non-homogeneous exposure of a thin elastic plate to a drop of favorable solvent, large shape changes can be induced, depending on the rate of swelling 7 . When the plate's thickness increases shape changes are localized to the swelling front and thus depend on the drop volume as well as the plate geometry ${ }^{8}$.

In this paper we investigate a model system consisting of a finite amount of liquid, namely a drop, placed on a swellable fibre. We propose a diffusive model, coupled with a description of the drop shape, that quantitatively describes the absorption dynamics.

\section{Absorption dynamics of a drop on a swelling fibre}

\subsection{Experiments}

Fibres of controlled properties are formed by moulding a degased liquid oligomer solution (polyvinylsiloxane (PVS), Zhermack Elite double 8,32 or polydimethylsiloxane (PDMS, Sylgard 184, Corning, PDMS to curing agent 10:1 ratio) into glass capillaries (inner radius $r_{0}=250,315$ and $475 \mu \mathrm{m}$ ) before extracting them after several hours to obtain elastomeric fibres by breaking the glass capillary and pulling gently on the fibers. No preliminary treatment is done to the glass. For PVS, the fibres' Young moduli $E=0.2$ (PVS8) and $0.9 \pm 0.1$ (PVS32) MPa and Poisson ratio $v_{P}=0.5$ are obtained by measuring the deflection of a clamped fibre under its own weight and with a simple stretching experiment. To ensure the fibre remains horizontal, we impose an initial tension on the fibres such that $\frac{L}{L_{0}}=1.15$, where $L_{0}=4 \pm 1 \mathrm{~cm}$ is the fibre length at rest, and keep this value constant for all experiments. When the fibre is stretched its radius decreases. We call $r_{s}$ the fibre radius in the stretched state and measure $r_{s}=233,293$ and $442 \mu \mathrm{m}$ with a variation of $\pm 20 \mu \mathrm{m}$ arising from errors in the measurement of the initial radius as well as variations in the tension from one experiment to another. All experiments are done at room temperature i.e. $20 \pm 1^{\circ} \mathrm{C}$. We place a drop, of varying volume $0.5<V<6 \mu \mathrm{L}$, of silicone oil (Carl Roth, M2, M3 and M5, of kinematic viscosities $v=2,3$ or $5 \mathrm{~mm}^{2} \cdot \mathrm{s}^{-1}$, respective densities 870,910 and $930 \mathrm{~kg} \cdot \mathrm{m}^{-3}$ and dynamic viscosities $\mu=2.3,3.3$ and $5.4 \mathrm{mPa} . \mathrm{s}$, and surface tension $0.018 \pm 0.001 \mathrm{~N} / \mathrm{m}$ measured with a pendant drop experiment), on the horizontal stretched fibre with 
(a)

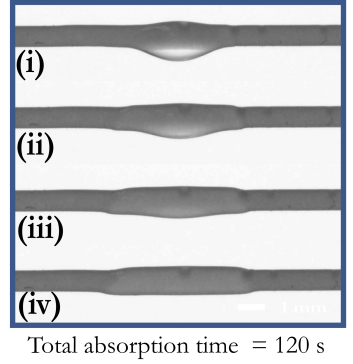

(c)
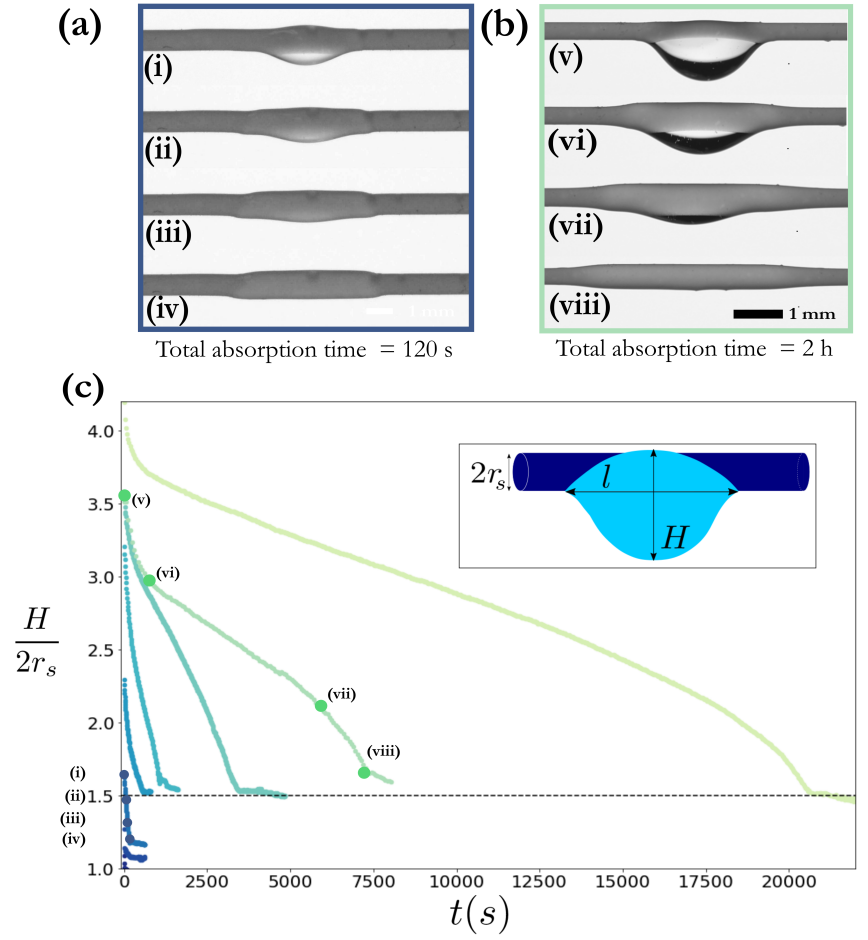

Fig. 1 Absorption dynamics of a drop on a swelling fibre.(a, b) Drop of silicone oil $\left(v=2 \mathrm{~mm}^{2} . \mathrm{s}^{-1}\right)$ sitting on a PVS32 fibre $\left(r_{s}=233 \mu \mathrm{m}\right)$ for initial volumes of 0.6 and $2 \mu \mathrm{L}$ respectively. Pictures are taken at 0 , 50,100 and $150 \mathrm{~s}$ for pictures (i) to (iv) and at $0,1200,6000,7000 \mathrm{~s}$ for pictures (v) to (viii). (c) Drop height $H$ in time on a swelling fibre for various initial volumes $(\mathrm{V}=0.05,0.1,0.5,1.4,3.2,4.1,3.9,5.7 \mu L)$. The height is always measured from drop apex to drop apex as shown in the inset.

a micropipette. Both PDMS and PVS spontaneously swell by absorbing the silicone oil. Pictures of the drop are taken at regular intervals using a Nikon D810 camera and a $105 \mathrm{~mm}$ macro lens. The characteristic evaporation time for a drop of $2 \mu \mathrm{L}$ on a fibre of radius $250 \mu \mathrm{m}, t_{\text {evap }} \simeq 80000$ s, is significantly larger than the duration of a typical experiment. We thus neglect evaporation effects.

The drop is absorbed by the fibre over time, leading to a localized swelling of the fibre (figures 1 (a) and (b)). Notations are defined in the inset of figure 1 (c). Depending on the initial volume of the drop, the time taken to absorb the liquid can vary from a few seconds to several hours. We distinguish two different situations reflected by the variations of the drop height $H$ with time (figure1 (c)).

For a small initial volume ( $V=0.6 \mu \mathrm{L}$, points (i)-(iv), blueframed pictures in figure 1 (a) and corresponding blue curve in figure 1 (c)), the fibre swells as the drop height decreases: the liquid is absorbed progressively into the fibre. The drop height $\frac{H}{2 r_{s}}$ decreases quickly to reach a plateau value slightly over 1 . The exact value of $H$ after absorbing the drop depends on how much the fibre has swollen locally. For a larger drop $(V=3.9 \mu \mathrm{L}$, points (v)(viii), green-framed pictures in figure 1 (b), and corresponding green curve in figure 1 (c)), we observe three different regimes. In the first few minutes (from (v) to (vi)), similarly to small drops, the drop height $H$ decreases quickly and the fibre radius at the position of the drop becomes larger. From (vi) to (vii), the fibre radius remains constant and the decrease of $H$ slows down significantly as the liquid diffuses slowly from the drop edges. In the third regime (from (vii) to (viii)), once most of the drop is absorbed within the fibre, the decrease of $H$ accelerates again until the drop is fully absorbed by the fibre. After point (viii), the drop is no longer visible: the fluid eventually diffuses within the fibre leading to a slow decrease of $H$.

These experiments show that a small increase in the initial drop volume can have a dramatic effect on the total absorption time. Indeed, a threefold increase in drop volume leads to an over hundred-fold increase in absorption time. We measure the total absorption time, $T_{a b s}$, a fibre takes to absorb a single droplet. This time is defined at the inflexion point of $\frac{H}{2 r_{s}}$ (points (iv) and (viii) in figure 11). We plot the total absorption time as a function of the initial drop volumes (figure 2). For small drops this time remains below a few minutes. For drops that are larger than $3 \mu L$, $T_{a b s}$ increases sharply with the drop volume. The slowing down of the absorption (between points (vi) and (vii) in figure 1) thus only happens for drops larger than a critical volume.

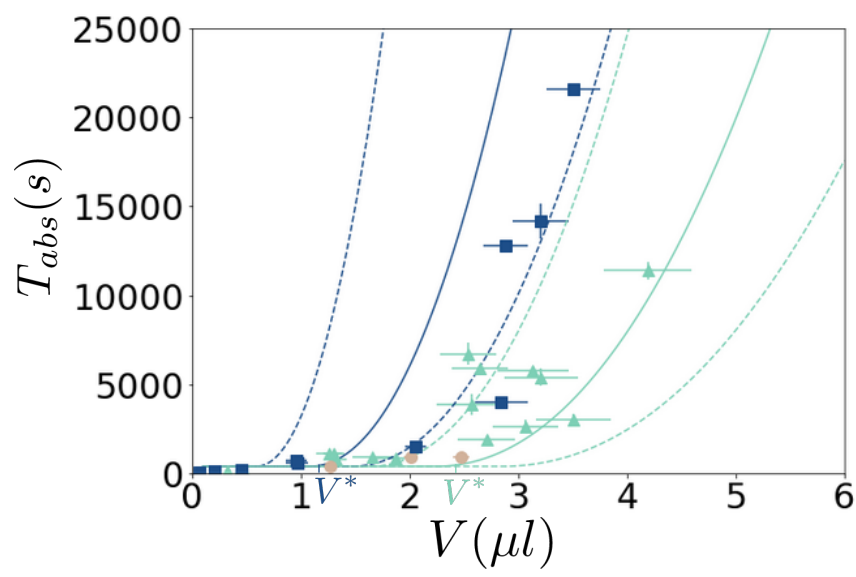

Fig. 2 Total absorption time as a function of the initial volume for various fibre radii (blue: $r_{s}=233 \mu \mathrm{m}$, green: $r_{s}=293 \mu \mathrm{m}$, brown: $r_{s}=442 \mu \mathrm{m}$ ). The lines are given by equation (14) obtained with the average measured radius $r_{s}$ and swelling ratio $\lambda_{\max }$ (plain line) and with the extreme values of the measured swelling ratios and radii (dashed lines). $V^{*}$ is the critical volume found theoretically as described in section 3.2 .

In the next sections, we build a model for the absorption dynamics, and demonstrate the existence of a critical volume that highly depends on the fibre radius and swelling properties. In order to construct a model of these dynamics, we first need to describe the drop geometry depending on its volume (section 2.2) as well as the swelling dynamics of a fibre immersed in a liquid bath (section 2.3).

\subsection{Drop geometry on a fibre without swelling and in the presence of gravity}

The shape of a drop on a fibre without gravity is well described and characterized both experimentally and analytically 910111112 . For fully wetting drops, the shape of the drop can be described analytically. In particular, using surface minimization and geometry 


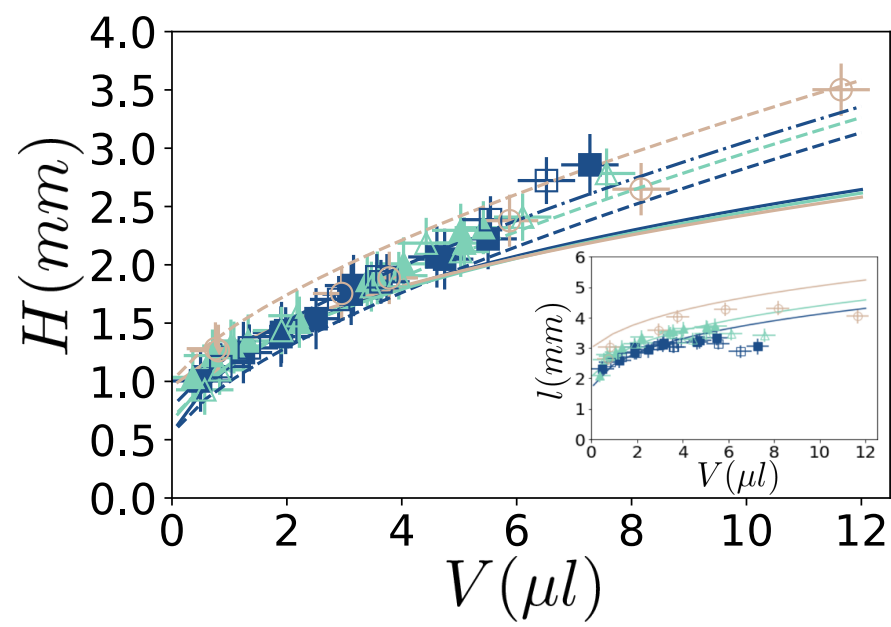

Fig. 3 Drop geometry on a fibre without swelling: Measured height and length (inset) of a drop of viscous silicone oil $\left(v=100 \mathrm{~mm}^{2} . \mathrm{s}^{-1}\right)$ for various fibre radii: $r_{s}=233$ (blue $\square$ ), 293 (green $\triangle$ ) and $442 \mu \mathrm{m}$ (brown $\circ$ ) with empty symbols, and on the same fibres pre-swollen overnight (filled symbols). Plain lines corresponds to the analytical prediction without gravity given by eqs. (1,2), and dashed lines to the empirical prediction given by eq. (4) for dry fibres (small dash, $r=r_{s}$ ) and pre-swollen fibres (dash-point, $r=1.5 \times r_{s}$ ).

arguments, Carroll ${ }^{11}$ derives equations relating the drop volume, length and height depending on the fibre radius:

$$
\begin{gathered}
V=\frac{2 \pi H}{3}\left[\left(2 r^{2}+3 r H+2 H^{2}\right) E(\chi)-r^{2} F(\chi)\right]-\pi r^{2} l \\
l=2\left[r F(\chi)+\frac{H}{2} E(\chi)\right]
\end{gathered}
$$

where

$$
\chi^{2}=\frac{H^{2}-R^{2}}{H^{2}}
$$

and $F(\chi)$ and $E(\chi)$ are complete elliptic integrals of the first and second kind. We can solve these equations numerically for $H$ and $l$ and obtain the curves shown in figure 3 . Less attention has been devoted to drops placed on fibres and subjected to gravitational effects 1314 . In our experiments, drops are deformed by gravity (as seen in figure 1) and we look for a relation between the drop height $H$ and length $l$ with its volume $V$, without any swelling. In order to have no swelling effect, we used silicone oil of same surface tension and wetting properties on the PVS, but high viscosity $\left(v=100 \mathrm{~mm}^{2} \cdot \mathrm{s}^{-1}\right.$ ) so that no swelling can be observed during our measurements of the drop shape. We have first verified that no swelling occurs over an immersion of 2 hours of PVS fibres in the high viscosity oil. Drops of high viscosity oil placed on a horizontal stretched fibre are thus at equilibrium. We weight the fibre and drop system with precision scales in order to accurately determine the drop volume (Mettler Toledo, precision: $0.0001 \mathrm{~g}$ ). Pictures are taken perpendicular to the fibre orientation in order to carefully measure the drop's height and length, which are then plotted for different liquid volumes (figure 3). The drop's height increases with the volume of the drop following the analytical prediction without gravity up to $V \approx 4 \mu L$, when gravity can no longer be neglected. For higher volumes, the drop shape is then modified by gravity: axisymmetry is lost and the drop stretches in the vertical direction. Indeed, when $V \geq 4 \mu L$, the drop height increases above the predicted value and its length reaches a plateau, i.e. gravity keeps the drop more compact in the horizontal direction. Moreover, for identical volumes, the drop length increases with the fibre radius. We use these experiments to find an empirical relationship between liquid volume and drop height $H$ in the form of

$$
H-2 r=a V^{b}
$$

While this relationship can be refined for every individual radius, $a=534 m^{-2}$ and $b=0.67$ give satisfactory results for all radii. In order to have the same conditions as in the swelling experiment, i.e. the same fibre radius and the same wetting condition, we also pre-swell fibres by immersing them overnight in a bath of low viscosity oil (viscosity $v=5 \mathrm{~mm}^{2} . \mathrm{s}^{-1}$ ), and similarly place single drops on them. The results are reported in figure 3 and we observe the same scaling for pre-swollen and dry fibres. There is thus no effect of swelling on the drop shape, i.e. swelling only changes the radius of the fibre. There is thus a unique drop shape for a given volume and fibre radius. We thus use the empirical relation (4) to describe the drops during the whole absorption experiment, i.e. to guess the exact volume of the drop at all times, using the measured swollen radius and drops's height $H$. Note that this relation depends on the contact angle between the liquid and the solid, as well as the surface tension and density of the fluid and has thus to be determined for each given material.

\subsection{Swelling dynamics of a fully immersed fibre}

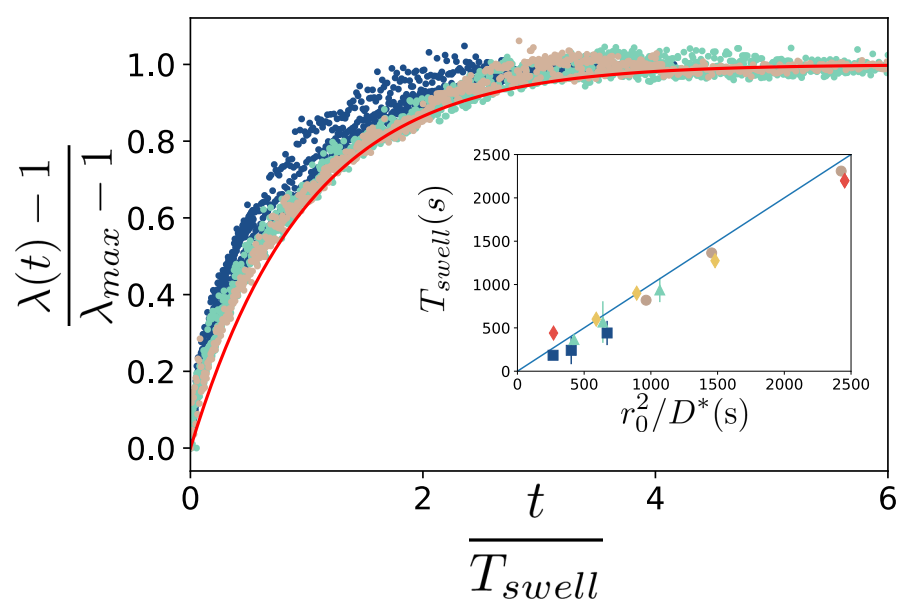

Fig. 4 Swelling dynamics of an immersed fibre. Evolution of the renormalized swelling ratio of a PVS fibre immersed in a bath of silicone oil ( $\mu=2.3 \mathrm{mPa}$.s (dark blue), $\mu=3.3 \mathrm{mPa}$.s (light blue) and $\mu=5.4$ $\mathrm{mPa}$.s (brown)) for various fibre radii $\left(r_{0}=250,315\right.$ and $\left.475 \mu \mathrm{m}\right)$. The red plain line corresponds to eq (6). The inset shows the value of $T_{\text {swell }}$ as a function of fibre radius and diffusion coefficient for PVS $\left(r_{0}=250\right.$ (blue squares), 315 (light blue triangles) and $475 \mu \mathrm{m}$ (brown circles)) and PDMS ( $r_{0}=475 \mu \mathrm{m}$, yellow diamonds).

We study the swelling dynamics of one fibre. In our experiments, the unsaturated fibre at the drop position is locally surrounded by oil. The liquid is first absorbed at high speed as can be observed from the rapid decrease in drop size during the first few minutes of the experiment (figure 1 (c)). To understand the 
swelling dynamics in this region, we measure the swelling rate over time of a fibre fully immersed in a bath of oil. We immerse single fibres of various radii $\left(r_{0}=250,315\right.$ and $\left.475 \mu \mathrm{m}\right)$ into a bath of silicone oil (of various viscosities $\mu=2.3,3.3$ and 5.4 $\mathrm{mPa} . \mathrm{s})$ and track their lengths and radii in time. The fibres are only clamped at one end to be held in place. The swelling is thus unconstrained. No significant difference between the swelling in the radial and in the axial direction is observed. We assume the swelling to be isotropic and measure the fibre's length $L$ as it increases with time and compare it to its initial length $L_{0}$.

We define the swelling ratio as

$$
\lambda(t)=\frac{L(t)}{L_{0}}=\frac{r(t)}{r_{0}} .
$$

Once the fibre is immersed into the oil, $\lambda$ increases until it reaches a plateau value between 1.43 and 1.55. Variations might come from slight variations in the composition of the fibre or straindependent effects that will be further discussed in section 4 . We define the maximal swelling ratio as $\lambda_{\max }=\frac{L(t=\infty)}{L_{0}}$. All the data collapse onto a single line by plotting the renormalized swelling ratio $\frac{\lambda(t)-1}{\lambda_{\max }-1}$ as a function of nondimensional time $\frac{t}{T_{\text {swel }}}$, where $T_{\text {swell }}$ is a measured characteristic swelling time (figure 4). This dynamics is similar to that observed for the swelling of various gels $5 / 15$. The swelling dynamics of fully immersed objects such as spheres ${ }^{4}$ or cylinders ${ }^{3}$ is generally modeled by describing the complete thermo-mechanical processes of a swelling polymer, or by using pseudo-diffusive models to describe small deformations ${ }^{16} 17$. Here, we describe the evolution of the renormalized swelling ratio with a simple model such that

$$
\frac{\lambda(t)-1}{\lambda_{\max }-1}=1-e^{\frac{-t}{T_{\text {swell }}}}
$$

A similar expression has been used to describe the hydration of dehydrated cylindrical spaghetti ${ }^{5}$. While $\lambda_{\max }$ is constant for a given fibre material, $T_{\text {swell }}$ strongly depends on the initial fibre radius and oil viscosity as shown in the inset figure 4 , such that

$$
T_{\text {swell }}=\frac{r_{0}^{2}}{D^{*}}
$$

where $D^{*}$ is a pseudo-diffusion coefficient that depends on the oil viscosity and material properties. Here, since we consider a fibre for which the radius is small compared to its length, the important length-scale to consider is the fibre radius $r_{0}$. We measure $T_{\text {swell }}$ for several fibre radii, fibre composition and oil viscosity (inset Fig. 4), and determine $D^{*}$ for each material and viscosity. We performed these experiments with two different kinds of PVS and PDMS. The characteristics of the different elastomers are detailed in table 1 .

\section{Total absorption time and complete absorption dynamics}

We investigate the complete dynamics of a drop absorption on a fibre. We can accurately describe the early time of the swelling dynamics observed in figure 1 with equation (6), as a drop of solvent deposited on a fibre locally acts similarly to a bath of fluid
Table 1 Values of E, and $D^{*}$ for two different batches of PVS (polyvinylsiloxane Zhermack Elite double 32 and 8) and PDMS (polydimethylsiloxane, Sylgard 184, Corning) depending on oil viscosity.

\begin{tabular}{llll}
\hline Material & $E(\mathrm{MPa})$ & $\mu(\mathrm{mPa} . \mathrm{s})$ & $D^{*}\left(\mathrm{~m}^{2} . \mathrm{s}^{-1}\right)$ \\
\hline \multirow{2}{*}{ PVS 8 } & \multirow{2}{*}{0.2} & 2.3 & $2.31 \cdot 10^{-10}$ \\
& & 3.3 & $1.54 \cdot 10^{-10}$ \\
& & 5.4 & $0.92 \cdot 10^{-10}$ \\
\hline \multirow{2}{*}{ PVS 32 } & \multirow{2}{*}{0.9} & 2.3 & $2.34 \cdot 10^{-10}$ \\
& & 3.3 & $1.55 \cdot 10^{-10}$ \\
& & 5.4 & $0.93 \cdot 10^{-10}$ \\
\hline \multirow{2}{*}{ PDMS } & \multirow{2}{*}{1} & 2.3 & $1.68 \cdot 10^{-10}$ \\
& & 3.3 & $1.11 \cdot 10^{-10}$ \\
& & 5.4 & $0.67 \cdot 10^{-10}$ \\
\hline
\end{tabular}

on a swelling fibre. Indeed, the Laplace pressure inside the drop $P_{\text {laplace }} \simeq \frac{\gamma}{H} \simeq 10 \mathrm{~Pa}$ is significantly smaller that the swelling driving pressures, i.e. the osmotic pressure $\frac{18}{,} \Pi_{\text {mix }} \simeq \frac{R T}{V_{\text {mol }}} \simeq 10^{6} \mathrm{~Pa}$, where $V_{m o l}$ is the molar volume of the solvent, and the pore pressure $\sim E \lambda^{3} \sim 10^{5} \mathrm{~Pa}$. We thus estimate that the increase of pressure inside the drop due to its interface curvature does not affect the swelling dynamics at early times. We indeed observe a rapid decrease of the height of the drop $\frac{H}{2 r_{s}}$ with a typical time of order of $(4 \pm 2) T_{\text {swell }}$.

We measure the fibre radius $r(t)$ close to the drop center during the experiments presented in the first section (figure 5 (a)). Since the drop is acting as a liquid lens, it is not possible to measure the fibre radius directly below the drop with accuracy; we thus measure the radius on the edge of the drop. From its initial value $r_{s}$, the radius increases quickly, following a dynamic close to the one found in section 2.3 (dashed blue line, eq. (6)), before reaching a plateau value. The time at which the plateau is reached corresponds to the end of the sharp decrease in drop size ((vi) figure 1 (c)). We note that here, the value of the swelling time is larger than the one of the immersed fibre $T_{\text {swell }}$ (about $4 T_{\text {swell }}$ ). This factor 4 might arise from the fact that we measure the radius on the side of the drop, which might differ from the radius below the drop. Furthermore, the model, obtained for fully immersed fibres, only accounts for radial diffusion, while axial diffusion from the drop edges might occur, slowing down the initial swelling. Provided that the volume is large enough, the radius reaches the maximal radius $r_{\max }=\lambda_{\max } r_{s}$. Indeed, we must define a critical volume $V_{\text {cap }}$ as the maximum fluid volume that can be absorbed by the fibre portion that is in contact with the drop at $t=0$, i.e.:

$$
V_{\text {cap }}=\left(\lambda_{\max }^{2}-1\right) \pi r_{s}^{2} l_{0}
$$

where $l_{0}$ is the drop length at $t=0$. This volume strongly depends on the geometry since the fibre radius also affects the drop length $l_{0}$. In fig. 5 (b), we plot the final swelling ratio $r\left(t=T_{a b s}\right) / r_{s}$ as a function of the drop volume compared to the critical volume. For small volumes, $V<V_{\text {cap }}, r\left(T_{a b s}\right) / r_{s}$ remains smaller than $\lambda_{\max }$, i.e. the initial volume is not sufficient to fully swell the fibre locally. For larger volumes, we reach a plateau value of $\lambda_{\max }=1.5$, i.e. the fibre reaches its maximum radius at the position of the drop during the experiment and the fibre is fully saturated with liquid below the drop. The transition occurs for $V / V_{\text {cap }} \gtrsim 1$. The sharp increase in absorption time corresponds to the volumes 

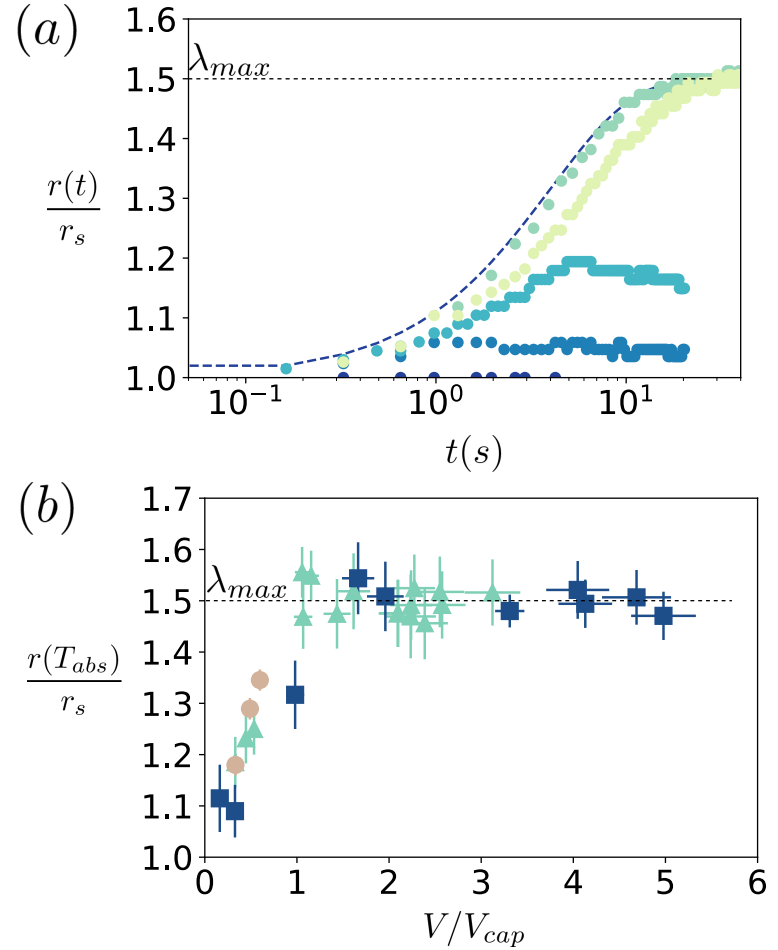

Fig. 5 (a) Evolution of the radius of the fibre beneath the drop for various initial drop volumes corresponding to the experiments shown in Fig. 1 with corresponding colors $\left(v=2 \mathrm{~mm}^{2} . \mathrm{s}^{-1}, \mathrm{PVS} 32, r_{s}=233 \mu \mathrm{m}\right.$, $V=0.05,0.1,0.5,1.4,4.1 \mu \mathrm{L}$ ). For high volumes (lighter curves) the fibre radius reaches its maximum value $r_{\max }=\lambda_{\max } r_{s}$ whereas for smaller volumes (darker curves), the swelling stops when there is no more fluid available in the drop. The dashed line corresponds to equation (6) with $T^{\prime}=4 T_{\text {swell }}$. (b) Final swelling ratio at the initial drop position vs. initial drop volume compared to $V_{\text {cap }}$ for various fibre radii: $r_{s}=233$ (blue $\square$ ), 293 (green $\triangle$ ) and $442 \mu \mathrm{m}$ (brown $\circ$ ).

at which the fibre is locally saturated with oil at the drop position.

\subsection{Model}

The localized saturation of the fibre is responsible for the large differences in total absorption times. We divide the dynamics into two different stages. At short times, the absorption dynamic is dominated by diffusion in the radial direction: the fibre locally swells and absorbs the oil quickly until reaching its maximal swelling ratio (saturation). Then, the dynamic is dominated by diffusion of the fluid within the fibre in the axial direction. We assume that at a given time $t$ the drop has a volume $V$ and the fibre has a radius $r$. Knowing $V$ and $r$ we calculate $H$ and $l$ (the drop length) using equation 4 and the notations presented in the schematic (figure 6 (a)). The portion of the fibre in contact with the drop at $t$ is a cylinder of radius $r$ and length $l$. We then calculate the decrease in drop volume that is directly associated to an increase in fibre radius: Assuming molecular incompressibility we can write that the variation in fibre volume $V^{f}$ is equal to the variation in drop volume, i.e.:

$$
\frac{\partial V}{\partial r}=-\frac{\partial V^{f}}{\partial r}
$$

The volume of the wetted fibre portion has a volume $V_{\text {wet }}=\pi r^{2} l$. Let us temporarly consider this wetted fibre portion as a cylindre that will swell isotropically (as from figure 6 (a) (ii) to (ii bis)). For mathematical consistency, we call $l_{\text {wet }}$ the length of the wetted cylindre, such that

$$
\frac{\partial V_{w e t}}{\partial r}=2 \pi r l_{w e t}+\pi r^{2} \frac{\partial l_{w e t}}{\partial r}
$$

Assuming that the swelling is isotropic, we have $\frac{\partial l_{\text {wet }}}{\partial r}=\frac{l_{\text {wet }}}{r}$ and thus

$$
\frac{\partial V^{f}}{\partial r}=\frac{\partial V_{w e t}}{\partial r}=3 \pi r l_{\text {wet }}=3 \pi r l
$$

$1(\mathrm{~V}, \mathrm{r})$ is given by equation (2). Using this relationship we can increment $r$ (until it reaches $r_{\max }$ ) and calculate $l, V$, and deduce $H$ for every step. Here, we use equation (2) to calculate $l$ which is satisfactory for small drops $(V<4 \mu L)$ as can be seen in the inset of figure 3. To reproduce the path taken by the drop in the $(V, H)$ space during the experiment, we divide the simulations into the two regimes described above (figure 6 (a)):

1. At first, the fibre radius increases from $r_{s}$ (i) to $\lambda_{t=\infty} r_{s}$ (ii). If $\lambda_{t=\infty}=\lambda_{\max }$ (iii), we proceed to the second step. If $\lambda_{t=\infty}<$ $\lambda_{\max }$, we stop. We estimate that the dynamics of this first phase (before saturation, i.e. going from a dry to a saturated fibre) is identical to the case of an immersed fibre, and thus occurs over a time close to $T_{\text {swell }}$.

2. If saturation is reached, we move on to the second phase. The fibre is saturated in fluid at the position of the drop. We define $\widetilde{V}$ as the remaining volume of the drop when saturation is reached (i.e. the point at which the upper curve is reached), which is calculated using our model eqs (9-11). The drop volume then reduces through diffusion in the axial direction of the fibre until it reaches 0 (iv).

In the second phase, we assume that the fluid diffuses inside the fibre in the axial direction with a typical diffusion coefficient $D^{*}$, whose value is the one deduced from our experiments with an immersed fibre. We thus have

$$
L_{\text {diff }}=\sqrt{D^{*} t},
$$

where $L_{\text {diff }}$ is the length over which the fluid has to move (figure 6 (a)). During this phase, we consider a constant droplet length. We calculate $L_{d i f f}$ by estimating the length of fibre necessary to absorb the remaining fluid $(\widetilde{V})$ once saturation is reached (in green, figure 6(a)). Since the fibre radius increases from $r_{s}$ to $\lambda_{\max } r_{s}$, we get the following:

$$
L_{\text {diff }}=\frac{\widetilde{V}}{2 \pi r_{s}^{2}\left(\lambda_{\max }^{2}-1\right)} .
$$

The factor $1 / 2$ comes from the fact that the fluid can diffuse from both sides of the drop. We can now obtain an estimation of $T_{a b s}$ as a sum of the time taken for the two phases, i.e. $T_{\text {swell }}$ (phase 1 ) and $L_{d i f f}^{2} / D^{*}$ (phase 2) :

$$
T_{a b s} \simeq T_{\text {swell }}+\frac{\widetilde{V}^{2}}{4 \pi^{2} D^{*} r_{s}^{4}\left(\lambda_{\max }^{2}-1\right)^{2}} .
$$




\subsection{Results}

We can now plot the evolution of the drop volume and drop height during the absorption for different initial volumes (figure 6 (b)). Our model (dashed lines) is in good agreement with the experimental data. The experiments always start on the lower curve $H(V)$ found empirically for a dry fibre (eq. (4) with $r=r_{s}$ ). The drop then gets absorbed by the fibre leading to a decrease of $V$ and $H$. The paths taken by the drops are given by the dashed lines. If the path reaches the upper curve, which corresponds to the curve $H(V)$ found empirically for a swollen fibre (eq. (4) with $r=r_{\max }$ ), the fibre is locally saturated. The drop then gets absorbed slowly by diffusion in the axial direction of the fibre and moves along the upper curve until it reaches $\frac{H}{2 r_{s}}=\lambda_{\max }$. We note that, as the time-step between two points is regular for each experiment $(\Delta t=60 s)$, their decreasing spacing thus indicate that the absorption speed decreases as we get closer to the upper curve corresponding to a locally saturated fibre. For small volumes, the upper curve is not reached and the fibre remains unsaturated. We can deduce the smallest initial volume for which the fibre is saturated $V^{*}$. We indicate this volume for two fibre radii in figure 2. $V^{*}$ is close to $V_{c a p}$ but also accounts for some of the diffusion occurring in the axial direction during the first phase of the experiment. For the experiments presented in figure 6 (b), we predict a critical volume $V^{*}=1.08 \mu L$; experimentally, we find a value close to $1.4 \mu \mathrm{L}$.

Our prediction of $T_{a b s}$ given by eq (14) is in good agreement with the experiments (Figure 2 (a)), i.e. our model captures the general behaviour of the system. Nevertheless, the predictions for this time are highly dependent on the swelling ratio $\lambda_{\max }$ and initial radius $r_{s}$ of the fibre. Dashed lines correspond to the extreme values of the measured swelling ratios and radii, which vary slightly in the experiments. The dependance of $T_{a b s}$ on $\lambda_{\max }^{2}$ and $r_{s}^{4}$ explains some of the variability of $T_{a b s}$. Moreover, the diffusive model is also an approximation in itself, and we do not account for variations in tension or fibre length. More precise calculations could be done using a full modeling of the swelling dynamics 19 , as will be discussed in the next section, but this is beyond the scope of this study.

\section{Discussion}

In this study we have established a quasi-diffusive model that accurately describes the absorption dynamics of a drop placed on a swellable fibre. In particular, it highlights the existence of a critical drop volume for which fibre saturation is reached below the drop, leading to a dramatic increase in the total absorption time. Porous but non swellable fibres should exhibit similar dynamics as liquid imbibition in any porous material also follows a pseudodiffusive law. However, the driving forces at play are not the same: capillary pressure in the pores induces motion of the wicking front whereas absorption is responsible for the swelling of the elastomer in our experiments. Chen et $\mathrm{al}^{20}$ studied the wicking kinetics of a liquid droplet into a porous yarn. The drop slowly wicks into the yarn without any changes in yarn radius. They observe that, for drops that saturate the yarn below the drop, the droplet is absorbed into the yarn in accordance with the diffusivelike Lucas-Washburn equation, and that the time of droplet absorption is a linear function of the initial droplet volume squared $V^{2}$, which is similar to the scaling of eq. (14), neglecting the initial radial penetration of liquid below the drop. However, the presence of swelling modifies the absorption kinetics strongly, as a $50 \%$ increase in fibre radius reduces the diffusion time by a factor 0.2 , since the fluid moves over a much shorter distance than without swelling. This effect is evidenced by the factor $\lambda_{\max }^{-4}$ in eq. (14).

Furthermore, some aspects of our model could be perfected by adding the detailed poroelastic features of our system. The swelling time that we found in section 2.3, corresponds to the typical poro-elastic time-scale $\frac{4 / 7 / 15 / 21}{2} r_{0}^{2} / D^{*}$, where $D^{*} \equiv E k / \mu, k$ being the permeability of the material. For most experiments, we use Zhermack Elite double 32 PVS for which $\mu D^{*}=4.67 \cdot 10^{-13}$ $\mathrm{kg}^{-1} \cdot \mathrm{m}^{-1}$, corresponding to a permeability of order $k \sim 10^{-18}$ $\mathrm{m}^{2}$ in agreement with previously reported results 722 . A different elastomer (Elite Double 8 PVS), which is both softer (lower $E$ ) and more permeable (higher $k$ ) actually has a similar constant $D^{*}$. We also perform experiments with PDMS, which is more rigid but more permeable and has a slightly smaller diffusion coefficient. Both experimental and theoretical studies ${ }^{23}$ show that the swelling ratio of a polymer (and thus in our case the final radius of the fibre) is dependent on the strain. In our experiments, we found the final radius to be very similar in both the unconstrained case of an immersed fibre (section 2.3) and in the case of a stretched fibre $(2)$, with some variability $( \pm 0.05)$ in $\lambda_{\max }$ from one experiment to another. Since swelling leads to a decrease in fibre tension, the final strain and thus the final radius at $t=T_{a b s}$ depends on the initial tension, the fibre length and the drop volume. The variation in fibre length might explain some of the dispersion observed in $\lambda_{\max }$ and in $T_{a b s}$. These effects might be of importance in further studies of fluids interacting with stretched fibres.

\section{Beating the saturation}

The cylindrical geometry of the fibre keeps the drop within a small portion of the fibre. In this case, although the drops are totally wetting, they will not spread but remain compact due to this specific shape. This particular characteristic prevents the rapid spreading and thus rapid absorption which is generally observed on a flat surface. The dynamics of absorption can be explained using the swelling dynamics of an immersed fibre and the drop geometry. Using simple diffusion arguments, we find that for drop volumes higher than a critical value dependent on the fibre radius, the total imbibition time increases dramatically, as the solvent diffuses slowly over a much larger distance in the fibre direction than only in the radial direction. To decrease this total absorption time it is critical to avoid local saturation of the fibre. Different strategies can be used to avoid saturation. Firstly, the drop can be forced to move e.g. by inclining the fibre or by varying the fibre geometry 24,25 . Secondly, one could force the drop to spread over a much larger distance. This can be achieved by using 
(a)
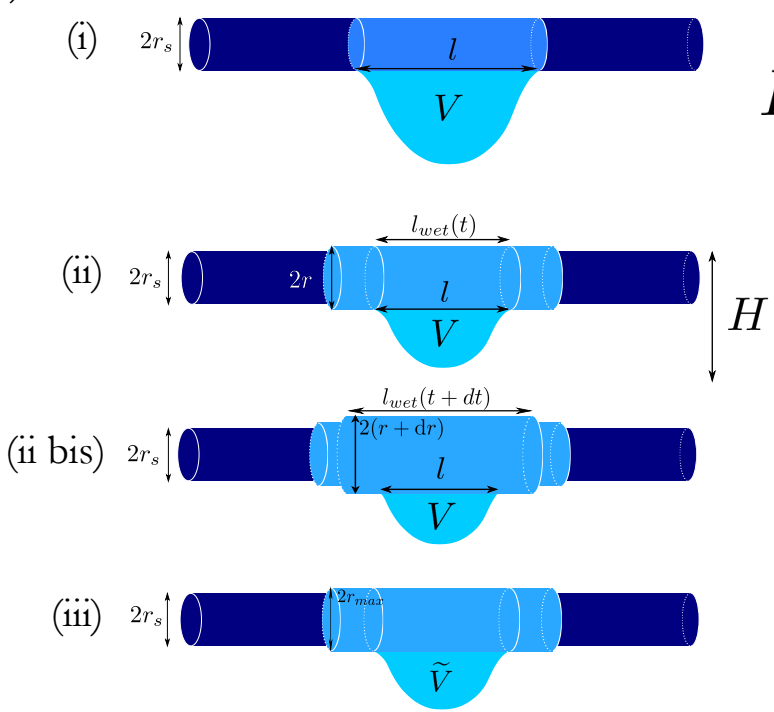

(iv)

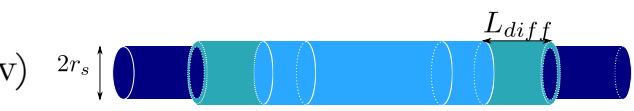

(b)

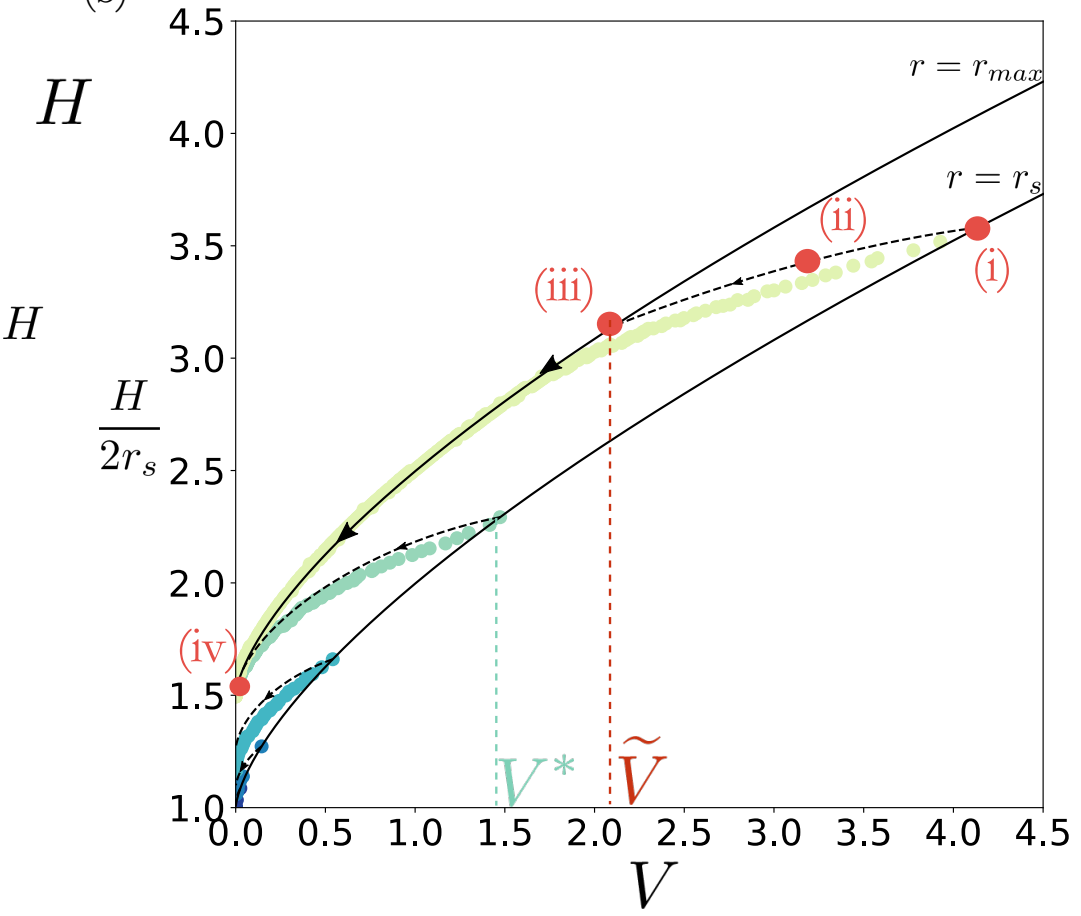

Fig. 6 (a) Notations for the absorption of a drop by a swelling fibre. (b) Evolution of drop volume and drop height $H(V)$ during the absorption corresponding to the experiments shown in Fig. 1 and 5 with corresponding colors $\left(v=2 \mathrm{~mm}^{2} . \mathrm{s}^{-1}, \mathrm{PVS} 32, r_{s}=233 \mu \mathrm{m}, V=0.05,0.1,0.5,1.4,4.1 \mu \mathrm{L}\right)$. The full lines represent the drop height vs. volume for a dry fibre (lower curve, eq. (4) with $r=r_{s}$ ) and a fully swollen fibre (upper curve, eq. (4) with $r=r_{\max }$ ). The trajectories (dashed lines) are predicted using the model presented in section 3.1 The arrows indicate the time evolution of $H(V)$.

several parallel fibres 26,27 : while the typical absorption time of isolated drops on fibres is of order of 10 hours, a drop deposited on two parallel fibres quickly wicks between them, and the total absorption time is reduced to a few seconds. These results are reminiscent of experiments recently reported on cotton fabrics ${ }^{28}$; while a drop spreading between the filaments of a yarn is absorbed in less that a millisecond, its absorption time on a single fibre is $1.6 \mathrm{~s}$, i.e. 5000 times higher. In general, the absorption time will strongly depend on the local geometry and liquid distribution and movement. The absorption dynamics will thus be particularly complex in fibrous networks, where the imbibition fronts are generally non uniform, and liquid may spread ahead of the wetting front in the form of precursors films along fibres or within yarns 29 33. The model experiments presented in this paper may provide a base to study more complex dynamics and morphological changes occuring in fibrous media during swelling. They are also a basic example of a localized swelling phenomenon occuring in a poroelastic material.

\section{Conflicts of interest}

There are no conflicts to declare.

\section{Acknowledgements}

CD acknowledges the Fondation de l'École polytechnique for funding through the Chaire professorale Jean Marjoulet.

\section{References}

1 M. Alava and K. Niskanen, Reports on Progress in Physics, 2006, 69, 669-723.
2 A. Patnaik, R. S. Rengasamy, V. K. Kothari and A. Ghosh, Textile Progress, 2006, 38, 1-105.

3 S. A. Chester and L. Anand, Journal of the Mechanics and Physics of Solids, 2011, 59, 1978-2006.

4 T. Bertrand, J. Peixinho, S. Mukhopadhyay and C. W. MacMinn, Physical Review Applied, 2016, 6, 064010.

5 M. Nobile, G. Buonocore, A. Panizza and G. Gambacorta, Journal of Food Science, 2003, 68, 1316-1323.

6 E. Reyssat and L. Mahadevan, EPL (Europhysics Letters), 2011, 93, 54001.

7 D. P. Holmes, M. RochÃl', T. Sinha and H. A. Stone, Soft Matter, 2011, 7, 5188.

8 A. Phadnis, K. C. Manning, I. Sanders, T. P. Burgin and K. Rykaczewski, Soft Matter, 2018, 14, 5869-5877.

9 N. K. Adam, J. of the Society of Dyers and Colourists, 1937, 11.

10 A. S. F.W. Minor, Textile Research Journal, 1959, 10.

11 B. Carroll, Journal of Colloid and Interface Science, 1976, 57, 488-495.

12 G. McHale and M. Newton, Colloids and Surfaces A: Physicochemical and Engineering Aspects, 2002, 206, 79-86.

13 R. de Ruiter, J. de Ruiter, H. B. Eral, C. Semprebon, M. Brinkmann and F. Mugele, Langmuir, 2012, 28, 1330013306.

14 T.-H. Chou, S.-J. Hong, Y.-E. Liang, H.-K. Tsao and Y.-J. Sheng, Langmuir, 2011, 27, 3685-3692.

15 H. Schott, Journal of Macromolecular Science, Part B, 1992, 
31, 1-09.

16 A. Pandey and D. P. Holmes, Soft Matter, 2013, 9, 5524-5528.

17 M. Doi, Journal of the Physical Society of Japan, 2009, 78, 052001.

18 P. J. Flory and J. Rehner, The Journal of Chemical Physics, 1943, 11, 521-526.

19 S. A. Chester and L. Anand, Journal of the Mechanics and Physics of Solids, 2010, 58, 1879-1906.

20 X. Chen, K. Kornev, Y. Kamath and A. Neimark, Textile Research Journal, 2001, 71, 862-869.

21 T. Tanaka and D. J. Fillmore, The Journal of Chemical Physics, 1979, 70, 1214-1218.

22 J. Mark, Polymer Data Handbook, Oxford University Press, 1999.

23 J. Yoon, S. Cai, Z. Suo and R. C. Hayward, Soft Matter, 2010, 6004-6012.

24 E. Lorenceau and D. QuÃ̂rrÃl', Journal of Fluid Mechanics, 2004, 510, 29-45.
25 J. McCarthy, D. Vella and A. A. CastrejÃşn-Pita, Soft Matter, 2019, 15, 9997-10004.

26 H. M. Princen, J. Colloid Interface Sci., 1970, 14.

27 C. Duprat and S. Protiâlíre, EPL (Europhysics Letters), 2015, 111, 56006.

28 K. Zhang, K. Fang, R. Xie, Y. Song, T. Z and X. Zhang, Cellulose, 2020, 27, 9725-9736.

29 R. J. Roberts, T. J. Senden, M. A. Knackstedt and M. B. Lyne, JOURNAL OF PULP AND PAPER SCIENCE, 2003, 29, 9.

30 J. Bico and D. QuÂlrrâl, Europhysics Letters (EPL), 2003, 61, 348-353.

31 M. Alava, M. Dubé and M. Rost, Advances in Physics, 2004, 53, 83-175.

32 L. Zhu, A. Perwuelz, M. Lewandowski and C. Campagne, Journal of Adhesion Science and Technology, 2008, 17.

33 M. Parada, P. Vontobel, R. M. Rossi, D. Derome and J. Carmeliet, Transport in Porous Media, 2017, 119, 611-632. 